\title{
One loop renormalization of the electroweak chiral Lagrangian with a light Higgs boson
}

\author{
Feng-Kun Guo, ${ }^{1}$ Pedro Ruiz-Femenía, ${ }^{2}$ and Juan José Sanz-Cillero ${ }^{3}$ \\ ${ }^{1}$ Helmholtz-Institut für Strahlen- und Kernphysik and Bethe Center for Theoretical Physics, \\ Universität Bonn, D-53115 Bonn, Germany and State Key Laboratory of Theoretical Physics, \\ Institute of Theoretical Physics, CAS, Beijing 100190, China \\ ${ }^{2}$ Physik Department T31, James-Franck-Strasse, Technische Universität München, \\ D-85748 Garching, Germany \\ ${ }^{3}$ Departamento de Física Teórica and Instituto de Física Teórica, IFT-UAM/CSIC, \\ Universidad Autónoma de Madrid, Cantoblanco, 28049 Madrid, Spain
}

(Received 6 July 2015; published 6 October 2015)

\begin{abstract}
We consider the general chiral effective action which parametrizes the nonlinear realization of the spontaneous breaking of the electroweak symmetry with a light Higgs boson and compute the one-loop ultraviolet divergences coming from Higgs and electroweak Goldstone fluctuations using the background field method. The renormalization of the divergences is carried out through operators of next-to-leading order in the chiral counting, i.e., of $\mathcal{O}\left(p^{4}\right)$. Being of the same order in power counting, the logarithmic corrections linked to these divergences can be as important as the tree-level contributions from the $\mathcal{O}\left(p^{4}\right)$ operators and must be accounted for in the phenomenological analysis of experimental data. Deviations in the $\mathcal{O}\left(p^{2}\right)$ (leading-order) couplings with respect to the Standard Model values, e.g., in the $h \rightarrow W W$ coupling, would generate contributions from the one-loop chiral logarithms computed in this work to a vast variety of observables, which do not have a counterpart in the conventional electroweak effective theory with a linearly transforming Higgs complex doublet.
\end{abstract}

DOI: $10.1103 /$ PhysRevD.92.074005

PACS numbers: 12.39.Fe, 12.60.Fr, 12.60.Rc

\section{INTRODUCTION}

The lack of experimental evidence of the new physics states predicted by many natural solutions to the electroweak (EW) symmetry breaking has brought an increasing interest in the analysis of possible deviations from the Standard Model (SM) using broader frameworks. Assuming there is a large energy gap between the EW scale $v=246 \mathrm{GeV}$ and the new physics scale, a description of the EW symmetry breaking (EWSB) in general terms is provided by an effective field theory (EFT) built from the presently known particle content, including a light Higgs scalar, and based on the spontaneous symmetry breaking pattern of the SM: $G=S U(2)_{L} \times S U(2)_{R}$ breaks down to the custodial group $H=S U(2)_{L+R}$, where $S U(2)_{L} \times U(1)_{Y} \subset G$ is gauged and the three would-be Goldstone bosons $\pi^{a}$ that arise from the spontaneous EWSB give mass to the $W^{ \pm}, Z$ in the unitary gauge.

The existence of an approximate custodial symmetry guarantees that the $\rho$-parameter corrections are small, the latter being originated from radiative corrections or (custodial-symmetry breaking) operators that are subleading in the EFT power counting [1]. Without loss of generality, the Higgs $h$ will be taken to be a singlet under the full group $G$ and the Goldstones are nonlinearly realized in our EFT approach [2]; linear models with a Higgs complex doublet $\Phi$ (e.g., the SM) are just a subset within the general class of nonlinear theories, as it is always possible to express $\Phi$ in terms of the singlet $h$ and the nonlinearly realized
Goldstones. Indeed, there is a vast variety of beyond-SM theories where the Higgs is a composite particle, typically a pseudo-Goldstone of some type, and shows the characteristic nonlinear interaction structure of this kind of particles (see the review [3]).

The framework described above shares many similarities with the low-energy limit of QCD, also ruled by the $S U(2)_{L} \times S U(2)_{R}$ chiral symmetry, and we can expect that well-known aspects from QCD are reproduced in the nonlinear EW chiral EFT. In particular, we are interested in this work in the relevant role of the EW chiral logarithms arising from radiative corrections. Their QCD analogues arising at one loop are in many cases as important as the tree-level contributions from higher dimension operators, due to the nonlinear structure of the Goldstone interactions (that is the case for instance in $\pi \pi$ scattering in the scalarisoscalar channel [4]).

Motivated by this fact, in this paper we study the radiative corrections from scalar boson loops (Higgs and EW Goldstones) within the framework of a nonlinear EW chiral Lagrangian including a light Higgs (ECLh). Reference [5] computed the ultraviolet (UV) divergence at next-to-leading order (NLO) in the linear Higgs EFT. The present work complements that analysis and provides the UV divergences and the renormalization for the nonlinear case at NLO in the low-energy chiral counting, i.e., at $\mathcal{O}\left(p^{4}\right)$, where $p$ denotes low-energy scales, either lightparticle masses or momenta. The ECLh contains all the SM particles and it is expected to describe their interaction at 
energies much below the cutoff of the EFT, $\Lambda_{\mathrm{ECLh}}$, given by either the scale at which one encounters a new state or the energy where loop corrections turn too large to validate a perturbative expansion, naively $4 \pi v \approx 3 \mathrm{TeV}$.

Based on dimensional analysis it is possible to organize a low-energy expansion of the amplitudes in powers of low-energy scales $p$ [6,7] and implement a chiral power counting in the nonlinear EFT Lagrangian [8-10]. Renormalization is then carried out order-by-order in the low-energy expansion [4,11-13].

The one-loop effective action shows a series of UV divergences of higher dimension that require the inclusion of new operators in the Lagrangian, which are NLO in the chiral counting. While the precise value of the NLO couplings depends on the underlying physics, their running is fully determined by the leading-order (LO) Lagrangian and its symmetry structure. Unless assumptions about the physics underlying the ECLh are taken, the theoretical predictions for data analyses of next runs at present and future colliders [14] must account in full generality for the tree-level contributions from NLO operators [10,15], the running and the associated NLO logarithms (that are provided in this paper), and the NLO loop finite pieces $[8,16-19]$, which vary from one observable to another.

\section{LOW-ENERGY EFFECTIVE THEORY}

At low energies the amplitudes can be organized in terms of a chiral expansion in powers of the low-energy scales $p$. The effective Lagrangian is organized as $[8,9,10]$

$$
\mathcal{L}=\mathcal{L}_{2}+\mathcal{L}_{4}+\cdots,
$$

where $\mathcal{L}_{n}$ contains terms of $\mathcal{O}\left(p^{n}\right)$. The LO Lagrangian reads $[10,15,20,21]$

$$
\begin{aligned}
\mathcal{L}_{2}= & \frac{v^{2}}{4} \mathcal{F}_{C}\left\langle u_{\mu} u^{\mu}\right\rangle+\frac{1}{2}\left(\partial_{\mu} h\right)^{2}-v^{2} V \\
& +\mathcal{L}_{\mathrm{YM}}+i \bar{\psi} \not \emptyset \psi-v^{2}\left\langle J_{S}\right\rangle
\end{aligned}
$$

where $\langle\ldots\rangle$ stands for the trace of $2 \times 2 \mathrm{EW}$ tensors, $\mathcal{L}_{\mathrm{YM}}$ is the Yang-Mills Lagrangian for the gauge fields, $D$ is the gauge covariant derivative acting on the fermions, and $J_{S}$ denotes the Yukawa coupling of the fermions to the Higgs and Goldstone fields defined below. The factors of $v$ in the normalization of some terms are introduced for later convenience. $\mathcal{F}_{C}, V$ and $J_{S}$ are functionals of $x=h / v$, and have Taylor expansions

$$
\begin{aligned}
\mathcal{F}_{C}[x] & =1+2 a x+b x^{2}+\cdots, \quad J_{S}[x]=\sum_{n} J_{S}^{(n)} x^{n} / n !, \\
V[x] & =m_{h}^{2}\left(\frac{1}{2} x^{2}+\frac{1}{2} d_{3} x^{3}+\frac{1}{8} d_{4} x^{4}+\cdots\right)
\end{aligned}
$$

given in terms of the constants $a, b, m_{h}$, etc. [10,15,20,21], with $J_{S}^{(n)}$ the $n$th derivative with respect to $h / v$.
Nonetheless, although we will not make any assumption on the value of these coefficients, it is important to mention that the measurements from LHC and indirect EW precision tests are found to be close the expected values in the SM, where the Higgs and the EW Goldstones conform a complex doublet. In the nonlinear realization of the spontaneous EWSB, the Goldstones are parametrized through the coordinates $\left(u_{L}, u_{R}\right)$ of the $S U(2)_{L} \times$ $S U(2)_{R} / S U(2)_{L+R}$ coset space [2], with the unitary matrices $u_{L, R}$ being functions of the Goldstone fields $\pi^{a}$ which enter through the building blocks

$$
\begin{aligned}
u_{\mu} & =i u_{R}^{\dagger}\left(\partial_{\mu}-i r_{\mu}\right) u_{R}-i u_{L}^{\dagger}\left(\partial_{\mu}-i \ell_{\mu}\right) u_{L}, \\
\Gamma_{\mu} & =\frac{1}{2} u_{R}^{\dagger}\left(\partial_{\mu}-i r_{\mu}\right) u_{R}+\frac{1}{2} u_{L}^{\dagger}\left(\partial_{\mu}-i \ell_{\mu}\right) u_{L}, \\
f_{ \pm}^{\mu \nu} & =u_{L}^{\dagger} \ell^{\mu \nu} u_{L} \pm u_{R}^{\dagger} r^{\mu \nu} u_{R},
\end{aligned}
$$

where $r_{\mu \nu}=\partial_{\mu} r_{\nu}-\partial_{\nu} r_{\mu}-i\left[r_{\mu}, r_{\nu}\right]$, and the left-hand counter part $\ell_{\mu \nu}$ is defined analogously. The tensor $J_{S}$ is defined as

$$
J_{S}=J_{Y R L}+J_{Y R L}^{\dagger}, \quad J_{Y R L}=-\frac{1}{\sqrt{2} v} u_{R}^{\dagger} \hat{Y} \psi_{R}^{\alpha} \bar{\psi}_{L}^{\alpha} u_{L},
$$

where $\psi_{R, L}=\frac{1}{2}\left(1 \pm \gamma^{5}\right) \psi$ and $\psi=(t, b)^{T}$ is the topbottom SM $S U(2)$ doublet. The summation over the Dirac index $\alpha$ in $\psi_{R}^{\alpha} \bar{\psi}_{L}^{\alpha}$ is assumed and its tensor structure under $G$ is left implicit. The $2 \times 2$ matrix $\hat{Y}[h / v]$ is a spurion auxiliary field, functional of $h / v$, which incorporates the fermionic Yukawa coupling, allowing the inclusion of explicit custodial symmetry-breaking terms [9].

The low-energy chiral counting of the building blocks is provided by the scaling $\left\{\partial_{\mu}, r_{\mu}, \ell_{\mu}, m_{h, W, Z, \psi}\right\} \sim \mathcal{O}(p)$, $\left\{\pi^{a} / v, u_{L, R}, h / v\right\} \sim \mathcal{O}\left(p^{0}\right)$, and $\psi / v \sim O\left(p^{1 / 2}\right) \quad[6,7]$. Accordingly, covariant derivatives must scale as the ordinary ones and $g, g^{\prime}, \hat{Y} \sim \mathcal{O}(p / v)$. Since this implies $\mathcal{F}_{C} \sim \mathcal{O}\left(p^{0}\right), u_{\mu} \sim \mathcal{O}(p)$ and $f_{ \pm}^{\mu \nu}, J_{Y R L}, J_{S}, V \sim \mathcal{O}\left(p^{2}\right)$, the LO Lagrangian in Eq. (2) is $\mathcal{O}\left(p^{2}\right)$ and the one-loop corrections are formally $\mathcal{O}\left(p^{4}\right)[6-10]$.

The transformations of the building blocks under $G$ are given by

$$
\begin{aligned}
h & \rightarrow h, \quad Y \rightarrow g_{R} Y g_{R}^{\dagger}, \quad u_{R / L} \rightarrow g_{R / L} u_{R / L} g_{h}^{\dagger}, \\
r_{\mu} & \rightarrow g_{R} r_{\mu} g_{R}^{\dagger}+i g_{R} \partial_{\mu} g_{R}^{\dagger}, \quad \ell_{\mu} \rightarrow g_{L} \ell_{\mu} g_{L}^{\dagger}+i g_{L} \partial_{\mu} g_{L}^{\dagger}, \\
\mathcal{O} & \rightarrow g_{h} \mathcal{O} g_{h}^{\dagger} \quad \text { for } \mathcal{O} \in\left\{u_{\mu}, J_{Y R L}, J_{S}, f_{ \pm}^{\mu \nu}\right\},
\end{aligned}
$$

where $g_{R / L} \in S U(2)_{R / L}$ and $g_{h} \in S U(2)_{L+R}$ [2].

The SM is recovered by setting $\mathcal{F}_{C}=(1+h / v)^{2}, V=$ $\frac{1}{4} \lambda v^{2}\left[(1+h / v)^{2}-1\right]^{2}$ and $\hat{Y}=(1+h / v)\left(y_{t} P_{+}+y_{b} P_{-}\right)$, defined in terms of the SM Yukawa coupling constants $y_{q}$ and the projectors $P_{ \pm}=\left(1 \pm \sigma^{3}\right) / 2$. Other SM fermion doublets and the flavor symmetry breaking between 
generations can be incorporated by adding in $J_{Y R L}$, an additional family index in the fermion fields, $\psi^{A}$, and promoting $\hat{Y}$ to a tensor $\hat{Y}^{A B}$ in the generation space [22].

In our analysis, $\ell_{\mu}, r_{\mu}, \hat{Y}$ are spurion auxiliary background fields that keep the invariance of the ECLh action under $G$. When evaluating physical matrix elements, custodial symmetry is then broken in the same way as in the SM, keeping only the gauge invariance under the subgroup $S U(2)_{L} \times U(1)_{Y} \subset G[10,11,15,20]$,

$$
\ell_{\mu}=-\frac{g}{2} W_{\mu}^{a} \sigma^{a}, \quad r_{\mu}=-\frac{g^{\prime}}{2} B_{\mu} \sigma^{3}, \quad \hat{Y}=\hat{y}_{t} P_{+}+\hat{y}_{b} P_{-},
$$

with $\hat{y}_{t, b}$ being functionals of $h / v$.

\section{EFFECTIVE ACTION AT ONE LOOP}

Our aim is to compute the one-loop UV divergences of the effective action by means of the background field method [23]. We choose $u_{L}=u_{R}^{\dagger}$ [24] and perform fluctuations of the scalar fields (Higgs and Goldstones) around the classical background fields $\bar{h}$ and $\bar{u}_{L, R}$, respectively, in a way analogous to Ref. [25],

$u_{R, L}=\bar{u}_{R, L} \exp \left\{ \pm i \mathcal{F}_{C}^{-1 / 2} \Delta /(2 v)\right\}, \quad h=\bar{h}+\epsilon$,

with $\Delta=\Delta^{a} \sigma^{a}$. Without any loss of generality we have introduced the factor $\mathcal{F}_{C}^{-1 / 2}$ in the exponent for later convenience; it will allow us to write down the secondorder fluctuation of the action in the canonical form [23].

To obtain the one-loop effective action within the background field method, one retains the quantum fluctuations $\vec{\eta}^{T}=\left(\Delta^{a}, \epsilon\right)$ up to quadratic order [23],

$$
\mathcal{L}_{2}=\mathcal{L}_{2}^{\mathcal{O}\left(\eta^{0}\right)}+\mathcal{L}_{2}^{\mathcal{O}\left(\eta^{1}\right)}+\mathcal{L}_{2}^{\mathcal{O}\left(\eta^{2}\right)}+\mathcal{O}\left(\eta^{3}\right)
$$

where $\mathcal{L}_{2}^{\mathcal{O}\left(\eta^{0}\right)}=\mathcal{L}_{2}\left[\bar{u}_{L, R}, \bar{h}\right]$. The tree-level effective action is equal to the action evaluated at the classical solution, $\int \mathrm{d}^{\mathrm{d}} \times \mathcal{L}^{\mathcal{O}\left(\eta^{0}\right)}$. The background field configurations correspond to the solutions of the classical equations of motion, defined by the vanishing of the linear term $\mathcal{L}_{2}^{\mathcal{O}(\eta)}$ for arbitrary $\vec{\eta}$. They read

$$
\begin{aligned}
\nabla^{\mu} u_{\mu} & =-2 J_{P} / \mathcal{F}_{C}-u_{\mu} \partial^{\mu}\left(\ln \mathcal{F}_{C}\right), \\
\partial^{2} h / v & =\frac{1}{4} \mathcal{F}_{C}^{\prime}\left\langle u_{\mu} u^{\mu}\right\rangle-V^{\prime}-\left\langle J_{S}^{\prime}\right\rangle,
\end{aligned}
$$

with $J_{P}=i\left(J_{Y R L}-J_{Y R L}^{\dagger}\right)$ and the covariant derivative $\nabla_{\mu} \cdot=\partial_{\mu}+\left[\Gamma_{\mu}, \cdot\right]$. Here and in the following, we abuse of the notation by writing the background fields $\bar{u}_{\mu}$ and $\bar{h}$ as $u_{\mu}$ and $h$ for conciseness.
The quadratic fluctuation $\mathcal{L}_{2}^{\mathcal{O}\left(\eta^{2}\right)}$ reads

$$
\begin{aligned}
\mathcal{L}^{\mathcal{O}\left(\Delta^{2}\right)}= & -\frac{1}{4}\left\langle\Delta \nabla^{2} \Delta\right\rangle+\frac{1}{16}\left\langle\left[u_{\mu}, \Delta\right]\left[u^{\mu}, \Delta\right]\right\rangle \\
& +\left[\frac{\mathcal{F}_{C}^{-\frac{1}{2}} \mathcal{K}}{8}\left(\frac{\partial^{2} h}{v}\right)+\frac{\Omega}{16}\left(\frac{\partial_{\mu} h}{v}\right)^{2}\right]\left\langle\Delta^{2}\right\rangle \\
& +\frac{1}{2 \mathcal{F}_{C}}\left\langle\Delta^{2} J_{S}\right\rangle, \\
\mathcal{L}^{\mathcal{O}\left(\epsilon^{2}\right)}= & -\frac{1}{2} \epsilon\left[\partial^{2}-\frac{1}{4} \mathcal{F}_{C}^{\prime \prime}\left\langle u_{\mu} u^{\mu}\right\rangle+V^{\prime \prime}+\left\langle J_{S}^{\prime \prime}\right\rangle\right] \epsilon, \\
\mathcal{L}^{\mathcal{O}(\epsilon \Delta)}= & -\frac{1}{2} \epsilon \mathcal{F}_{C}^{\prime}\left\langle u_{\mu} \nabla^{\mu}\left(\mathcal{F}_{C}^{-\frac{1}{2}} \Delta\right)\right\rangle+\mathcal{F}_{C}^{-\frac{1}{2}} \epsilon\left\langle\Delta J_{P}^{\prime}\right\rangle,
\end{aligned}
$$

in terms of $\mathcal{K}=\mathcal{F}_{C}^{-1 / 2} \mathcal{F}_{C}^{\prime} \quad$ and $\quad \Omega=2 \mathcal{F}_{C}^{\prime \prime} /$ $\mathcal{F}_{C}-\left(\mathcal{F}_{C}^{\prime} / \mathcal{F}_{C}\right)^{2}$. Through a proper definition of the differential operator $d_{\mu} \vec{\eta}=\partial_{\mu} \vec{\eta}+Y_{\mu} \vec{\eta}$, one can rewrite $\mathcal{L}_{2}^{\mathcal{O}\left(\eta^{2}\right)}$ in the canonical form,

$$
\mathcal{L}_{2}^{\mathcal{O}\left(\eta^{2}\right)}=-\frac{1}{2} \vec{\eta}^{T}\left(d_{\mu} d^{\mu}+\Lambda\right) \vec{\eta}
$$

where $d_{\mu}$ and $\Lambda$ depend on $h, u_{L, R}$ and on the gauge boson and fermion fields. Explicit expressions for the quantities in Eq. (12) can be found in the Appendix.

The $\mathcal{L}_{2}^{\mathcal{O}\left(\eta^{2}\right)}$ term in the generating functional is just a Gaussian integral, which provides the one-loop effective action $[23,26]$,

$$
S^{1 \ell}=\frac{i}{2} \operatorname{tr} \log \left(d_{\mu} d^{\mu}+\Lambda\right),
$$

where "tr" stands for the full trace of the operator, also in coordinate space. One can then extract the residue of the $1 /(d-4)$ pole in dimensional regularization using the heat-kernel expansion [23],

$$
\begin{aligned}
S^{1 \ell} & =-\lambda \int \mathrm{d}^{\mathrm{d}} \mathrm{x} \operatorname{Tr}\left\{\frac{1}{12} Y_{\mu \nu} Y^{\mu \nu}+\frac{1}{2} \Lambda^{2}\right\}+\text { finite } \\
& =-\lambda \int \mathrm{d}^{\mathrm{d}} \mathrm{x} \sum_{k} \Gamma_{k} \mathcal{O}_{k}+\text { finite }
\end{aligned}
$$

with $\lambda=\left[16 \pi^{2}(d-4)\right]^{-1} \mu^{d-4}$. The divergence is determined by the nonderivative quadratic fluctuation $\Lambda$ and the differential operator $d_{\mu}$ through $Y_{\mu \nu}=$ $\left[d_{\mu}, d_{\nu}\right]=\partial_{\mu} Y_{\nu}-\partial_{\nu} Y_{\mu}+\left[Y_{\mu}, Y_{\nu}\right]$, and we note that both $\Lambda$ and $Y_{\mu \nu}$ are $\mathcal{O}\left(p^{2}\right)$. In Eq. (14) "Tr" refers to the trace over the $4 \times 4$ operators that act on the fluctuation vector $\vec{\eta}$. The basis of local operators $\mathcal{O}_{k}$ that covers the space of oneloop divergences contains purely bosonic terms (given in Table I) and operators including fermions [discussed later in Eq. (16)]. For the UV-divergent part of the effective action we have a chiral expansion in powers of $p$ similar to that in (1): $\mathcal{L}^{1 \ell, \infty}=\mathcal{L}_{2}^{1 \ell, \infty}+\mathcal{L}_{4}^{1 \ell, \infty}+\cdots$. 
TABLE I. Purely bosonic operators needed for the renormalization of the NLO effective Lagrangian $\mathcal{L}_{4}$. In the last column, we provide the first term $\Gamma_{k, 0}$ in the expansion of the $\Gamma_{k}$ in powers of $(h / v)$ by using $\mathcal{F}_{C}=1+2 a h / v+b h^{2} / v^{2}+\mathcal{O}\left(h^{3}\right)$. The first five operators $\mathcal{O}_{i}$ have the structure of the respective $a_{i}$ Longhitano operator [11,12] (with $i=1 \ldots 5$ ). In addition, $c_{6}=\mathcal{F}_{D 7}, c_{7}=\mathcal{F}_{D 8}$ and $c_{8}=$ $\mathcal{F}_{D 11}$ in the notation of Ref. [10]. The last operator of the list, $\mathcal{O}_{10}=2\left\langle r_{\mu \nu} r^{\mu \nu}+\ell_{\mu \nu} \ell^{\mu \nu}\right\rangle$, only depends on the EW field strength tensors and its coefficient is labeled as $c_{10}=H_{1}$ in the notation of Ref. [4].

\begin{tabular}{lccc}
\hline \hline$c_{k}$ & Operator $\mathcal{O}_{k}$ & $\Gamma_{k}$ & $\Gamma_{k, 0}$ \\
\hline$c_{1}$ & $\frac{1}{4}\left\langle f_{+}^{\mu \nu} f_{+\mu \nu}-f_{-}^{\mu \nu} f_{-\mu \nu}\right\rangle$ & $\frac{1}{24}\left(\mathcal{K}^{2}-4\right)$ & $-\frac{1}{6}\left(1-a^{2}\right)$ \\
$\left(c_{2}-c_{3}\right)$ & $\frac{i}{2}\left\langle f_{+}^{\mu \nu}\left[u_{\mu}, u_{\nu}\right]\right\rangle$ & $\frac{1}{24}\left(\mathcal{K}^{2}-4\right)$ & $-\frac{1}{6}\left(1-a^{2}\right)$ \\
$c_{4}$ & $\left\langle u_{\mu} u_{\nu}\right\rangle\left\langle u^{\mu} u^{\nu}\right\rangle$ & $\frac{1}{96}\left(\mathcal{K}^{2}-4\right)^{2}$ & $\frac{1}{6}\left(1-a^{2}\right)^{2}$ \\
$c_{5}$ & $\left\langle u_{\mu} u^{\mu}\right\rangle^{2}$ & $\frac{1}{192}\left(\mathcal{K}^{2}-4\right)^{2}+\frac{1}{128} \mathcal{F}_{C}^{2} \Omega^{2}$ & $\frac{1}{8}\left(a^{2}-b\right)^{2}+\frac{1}{12}\left(1-a^{2}\right)^{2}$ \\
$c_{6}$ & $\frac{1}{v^{2}}\left(\partial_{\mu} h\right)\left(\partial^{\mu} h\right)\left\langle u_{\nu} u^{\nu}\right\rangle$ & $\frac{1}{16} \Omega\left(\mathcal{K}^{2}-4\right)-\frac{1}{96} \mathcal{F}_{C} \Omega^{2}$ & $-\frac{1}{6}\left(a^{2}-b\right)\left(7 a^{2}-b-6\right)$ \\
$c_{7}$ & $\frac{1}{v^{2}}\left(\partial_{\mu} h\right)\left(\partial_{\nu} h\right)\left\langle u^{\mu} u^{\nu}\right\rangle$ & $\frac{1}{24} \mathcal{F}_{C} \Omega^{2}$ & $\frac{2}{3}\left(a^{2}-b\right)^{2}$ \\
$c_{8}$ & $\frac{1}{v^{4}}\left(\partial_{\mu} h\left(\partial^{\mu} h\right)\left(\partial_{\nu} h\right)\left(\partial^{\nu} h\right)\right.$ & $\frac{3}{32} \Omega^{2}$ & $\frac{3}{2}\left(a^{2}-b\right)^{2}$ \\
$c_{9}$ & $\frac{1}{24} \mathcal{F}_{C}^{\prime} \Omega$ & $-\frac{1}{3} a\left(a^{2}-b\right)$ \\
$c_{10}$ & $\frac{\left(\partial_{\mu} h\right)}{v}\left\langle f_{-}^{\mu \nu} u_{\nu}\right\rangle$ & $-\frac{1}{48}\left(\mathcal{K}^{2}+4\right)$ & $-\frac{1}{12}\left(1+a^{2}\right)$ \\
\hline \hline
\end{tabular}

The UV divergences with the structure of the $\mathcal{L}_{2}$ operators in Eq. (2) are

$$
\begin{aligned}
\mathcal{L}_{2}^{1 \ell, \infty}= & -\lambda\left\{\frac{1}{8}\left[\frac{\mathcal{F}_{C}^{\prime} V^{\prime}}{\mathcal{F}_{C}}\left(4-\mathcal{K}^{2}\right)-\mathcal{F}_{C} \Omega V^{\prime \prime}\right]\left\langle u_{\mu} u^{\mu}\right\rangle\right. \\
& -\frac{3 \mathcal{F}_{C}^{\prime} V^{\prime} \Omega}{8 \mathcal{F}_{C}}\left(\frac{\partial_{\mu} h}{v}\right)^{2}+\left[\frac{1}{2}\left(V^{\prime \prime}\right)^{2}+\frac{3 \mathcal{K}^{2}}{8 \mathcal{F}_{C}}\left(V^{\prime}\right)^{2}\right] \\
& \left.+\left(V^{\prime \prime}\left\langle J_{S}^{\prime \prime}\right\rangle-\frac{3 \mathcal{F}_{C}^{\prime} V^{\prime}}{2 \mathcal{F}_{C}}\left\langle\Gamma_{S}\right\rangle\right)\right\},
\end{aligned}
$$

where $\Gamma_{S}=\mathcal{F}_{C}^{-1}\left(J_{S}-\mathcal{F}_{C}^{\prime} J_{S}^{\prime} / 2\right)$. These UV divergences are canceled out through the renormalization of various parts of $\mathcal{L}_{2}$ : the couplings in the $\mathcal{F}_{C}$ term (1st line); the Higgs kinetic term (1st term in 2nd line), which requires a NLO Higgs field redefinition; the coefficients of the Higgs potential, e.g., the Higgs mass (2nd bracket in 2nd line); and the Yukawa term couplings in $Y$ (3rd line).

The $\mathcal{O}\left(p^{4}\right)$ divergences $\mathcal{L}_{4}^{1 \ell, \infty}$ are split here into two types, according to whether they include fermion fields or not. The purely bosonic $\mathcal{O}\left(p^{4}\right)$ divergences $\left.\mathcal{L}_{4}^{1 \ell, \infty}\right|_{\text {bos }}$ are summarized in Table I.

The structure of $\mathcal{O}\left(p^{4}\right)$ UV divergences with fermion operators is slightly more involved:

$$
\begin{aligned}
\left.\mathcal{L}_{4}^{1 \ell, \infty}\right|_{\text {ferm }}= & -\lambda\left\{\left\langle\left(\frac{\mathcal{K}^{2}}{4}-1\right) \Gamma_{S}-\frac{\mathcal{F}_{C} \Omega}{8} J_{S}^{\prime \prime}\right\rangle\left\langle u^{\mu} u_{\mu}\right\rangle\right. \\
& +\frac{3}{4} \Omega\left\langle\Gamma_{S}\right\rangle\left(\frac{\partial_{\mu} h}{v}\right)^{2}+\frac{1}{2} \Omega\left\langle\Gamma_{P} u^{\mu}\right\rangle\left(\frac{\partial_{\mu} h}{v}\right) \\
& \left.+\frac{1}{2}\left\langle J_{S}^{\prime \prime}\right\rangle^{2}+\frac{3}{2}\left\langle\Gamma_{S}\right\rangle^{2}+\frac{1}{\mathcal{F}_{C}}\left(2\left\langle\Gamma_{P}^{2}\right\rangle-\left\langle\Gamma_{P}\right\rangle^{2}\right)\right\},
\end{aligned}
$$

with $\Gamma_{P}=J_{P}^{\prime}-\mathcal{F}_{C}^{-1} \mathcal{F}_{C}^{\prime} J_{P} / 2$.

Any operator not listed in Eqs. (15) and (16) and Table I is not renormalized at one loop by scalar boson loops. In the
SM limit one finds $\Omega=0, \mathcal{K}=2$ and $J_{S}^{\prime \prime}=\Gamma_{S, P}=0$, so all the $\mathcal{L}_{4}^{1 \ell, \infty}$ operators in Eq. (16) and Table I vanish except $\Gamma_{10}^{\mathrm{SM}}=-1 / 6$, which turns out to be independent of the Higgs field and is absorbed through the renormalization of $g$ and $g^{\prime}$ in $\mathcal{L}_{\mathrm{YM}}$. Furthermore, apart of $\mathcal{L}_{\mathrm{YM}}$, only the nonderivative operators (the Yukawa term and Higgs potential) get renormalized due to the scalar loops in the SM limit.

\section{RENORMALIZATION}

In order to have a finite one-loop effective action the divergences in Eq. (14) are canceled by the counterterms

$$
\mathcal{L}^{\mathrm{ct}}=\sum_{k} c_{k} \mathcal{O}_{k}
$$

with the renormalization condition $c_{k}=c_{k}^{r}+\lambda \Gamma_{k}$.

The $\Gamma_{k}$ 's have a Taylor expansion of the form $\Gamma_{k}[h / v]=\sum_{n} \Gamma_{k, n}(h / v)^{n} / n$ !, and similarly, $c_{k}[h / v]=$ $\sum_{n} c_{k, n}(h / v)^{n} / n$ !. This leads to the renormalization group equations for the $\mathcal{O}\left(p^{4}\right)$ coefficients,

$$
\frac{\partial c_{k, n}^{r}}{\partial \ln \mu}=-\frac{\Gamma_{k, n}}{16 \pi^{2}} .
$$

Physically, this means that the NLO effective couplings will appear in the amplitudes in combinations with logarithms of energy scales $E$ in the form

$$
\mathcal{M}_{\mathcal{O}\left(p^{4}\right)} \propto\left(c_{k, n}^{r}(\mu)-\frac{\Gamma_{k, n}}{16 \pi^{2}} \ln \frac{E}{\mu}\right) E^{4} .
$$

As is well known from chiral perturbation theory, the size of these logs is not known a priori and can even be more dominant than the $\mathcal{O}\left(p^{4}\right)$ finite pieces. For instance, in QCD for $\mu \sim M_{\rho}$, the logarithms in the pion vector form factor are numerically subdominant in comparison with the 
tree-level contributions, whereas one finds the opposite behavior in $\pi \pi$ production in the scalar-isoscalar channel. There is a priori no reason to neglect the running of the coefficients in the ECLh Lagrangian when confronting the experimental data against the (nonlinear) effective description of EWSB.

In the last column of Table I we have written the contribution to $\Gamma_{k}$ that renormalizes the bosonic terms in $\mathcal{L}_{4}$ with lowest number of Higgs fields, $\Gamma_{k, 0}=\Gamma_{k}[0]$, which we have used as a check of our results. In the $\mathcal{F}_{C}=1$ limit ( $a=b=0)$, we recover the running of the Higgsless EW chiral Lagrangian [11,12]. Part of our results for the oneloop running have been already determined in the general ECLh case in $W W, Z Z, h h$ scattering $\left(c_{4,0}, \ldots, c_{8,0}\right)$ [19] and $\gamma \gamma$ scattering and related photon processes $\left(c_{1,0}\right.$, $c_{2,0}-c_{3,0}$ ) [8]. Although the corresponding experimental analyses are limited so far by statistics and yield very loose constraints on these couplings [27-29], their accurate determination or the feasibility to set more stringent bounds in the future requires a careful control of these $\mathcal{O}\left(p^{4}\right)$ loop corrections.

Since the operators proportional to $A_{\mu \nu} A^{\mu \nu}$ and $A_{\mu \nu} Z^{\mu \nu}$ are only contained in the combination $\Delta \mathcal{L}=$ $\left(c_{1} / 4+c_{10} / 2\right)\left\langle f_{+}^{\mu \nu} f_{+\mu \nu}\right\rangle \subset \mathcal{L}_{4}$, the NLO couplings that describe the vertices $h \rightarrow \gamma \gamma$ and $h \rightarrow \gamma Z$ are renormalization group invariant, as found in [8] and [16], respectively. As $\Gamma_{1} / 4+\Gamma_{10} / 2=-1 / 12$ is independent of $h$, a similar thing applies to $\gamma \gamma$ and $\gamma Z$ vertices with more Higgs fields $(\gamma \gamma, \gamma Z \rightarrow h h, h h h \ldots)$.

Deviations from the SM at LO (e.g., by $a \neq 1$ in $\mathcal{F}_{C}$, that modifies the $h W W$ coupling) would also imply the appearance of the four-fermion UV divergences in Eq. (16), and thus the contribution of the associated chiral logarithms to flavor-changing neutral current processes.

Let us finally observe that the Higgs potential gets divergent corrections proportional to $\left(V^{\prime}\right)^{2}$ and $\left(V^{\prime \prime}\right)^{2}$, i.e., proportional to $m_{h}^{4}$, that could be relevant in the study of the stability of the Higgs potential.

This paper has been focused on the one-loop contributions of SM scalar particles and the induced renormalization at NLO in the chiral counting. Because scalars couple derivatively in nonlinear EW models, the scalar loops are the only source of NLO divergences that scale like the fourth power of the external momenta, $\left(q_{i}\right)^{4}$, e.g., $c_{8}$ in Table I. The fermionic operators in $\left.\mathcal{L}_{4}^{1 \ell, \infty}\right|_{\text {ferm }}$ in (16) scale as $\left(q_{i}\right)^{3}$ and $\left(q_{i}\right)^{2}$ (recall that the fermion field scales as the square root of the external momenta $[7,9,10]$ ), with the remaining powers of $p$ given by the fermion masses. contributions from gauge bosons and fermions inside the loop, which are not included in this work, will produce UV divergences of order $\left(q_{i}\right)^{3}$ and $\left(q_{i}\right)^{2}$, as these particles couple nonderivatively and proportionally to the $g$ and $g^{\prime}$ gauge couplings and Yukawas $y_{t, b}$ (i.e., proportionally to the gauge boson and fermion masses.) The computation and analysis of the latter is postponed to future work.

\section{ACKNOWLEDGMENTS}

We would like to thank J. F. Donoghue, M. J. Herrero and A. Pich for discussions on the heat-kernel expansion and EW chiral Lagrangians and S. Saa for pointing out the simplification in the structure of the Higgs kinetic term. P. R. F. thanks P. Ruiz-Torres for stimulating and cheerful discussions. The work of F. K. G. is supported in part by the NSFC and DFG through funds provided to the SinoGerman CRC 110 "Symmetries and the Emergence of Structure in QCD" (NSFC Grant No. 11261130311) and NSFC (Grant No. 11165005). The work of J. J. S. C. is supported by ERDF funds from the European Commission [Grants No. FPA2010-17747, No. FPA2013-44773-P, No. SEV-2012-0249, and No. CSD2007-00042]. The research of P.R.F. was supported by the Munich Institute for Astro- and Particle Physics (MIAPP) of the DFG cluster of excellence "Origin and Structure of the Universe".

\section{APPENDIX: FURTHER DETAILS ON THE QUADRATIC FLUCTUATION}

The quadratic fluctuation of the LO Lagrangian obtained in Eq. (12) is provided here in detail. The derivative operator $d_{\mu}=\partial_{\mu}+Y_{\mu}$ is given by

$$
\begin{aligned}
Y_{\mu} & =\left(\begin{array}{cc}
\gamma_{\mu} & \frac{1}{2} \vec{b}_{\mu} \\
-\frac{1}{2} \vec{b}_{\mu}^{T} & 0
\end{array}\right), \\
\left(\gamma_{\mu}\right)_{i j} & =-\frac{1}{2}\left\langle\left[\sigma^{i}, \sigma^{j}\right] \Gamma_{\mu}\right\rangle, \\
\left(\vec{b}_{\mu}\right)_{k} & =-\frac{1}{2} \mathcal{F}_{C}^{\prime} g_{C}\left\langle u_{\mu} \sigma^{k}\right\rangle,
\end{aligned}
$$

with $g_{C} \equiv \mathcal{F}_{C}^{-1 / 2}$ and the indices $i, j, k$ taking values from 1 to 3 .

The second contribution in the quadratic fluctuation of the LO Lagrangian (12) is provided by the $4 \times 4$ matrix $\Lambda$, which has the form

$$
\begin{aligned}
\Lambda= & \left(\begin{array}{cc}
\left(\sigma+\frac{1}{4} \vec{b}_{\mu} \vec{b}_{\mu}^{T}\right) & \left(-\vec{a}+\frac{1}{2} d^{\mu} \vec{b}_{\mu}\right) \\
\left(-\vec{a}+\frac{1}{2} d^{\mu} \vec{b}_{\mu}\right)^{T} & \left(\omega+\frac{1}{4} \vec{b}_{\mu}^{T} \vec{b}_{\mu}\right)
\end{array}\right), \\
\sigma_{i j}= & \left(-\frac{1}{2}\left(\partial^{2} \ln \mathcal{F}_{C}\right)-\frac{1}{4}\left(\partial_{\mu} \ln \mathcal{F}_{C}\right)^{2}\right) \delta_{i j} \\
& -\frac{1}{8}\left\langle\left[u_{\mu}, \sigma^{i}\right]\left[u^{\mu}, \sigma^{j}\right]\right\rangle-\mathcal{F}_{C}^{-1}\left\langle J_{S}\right\rangle \delta^{i j}, \\
\omega= & -\frac{1}{4} \mathcal{F}_{C}^{\prime \prime}\left\langle u_{\mu} u^{\mu}\right\rangle+V^{\prime \prime}+\left\langle J_{S}^{\prime \prime}\right\rangle, \\
(\vec{a})_{k}= & -\frac{1}{2} \mathcal{F}_{C}^{\prime}\left(\partial^{\mu} g_{C}\right)\left\langle u_{\mu} \sigma^{k}\right\rangle+g_{C}\left\langle J_{P}^{\prime} \sigma^{k}\right\rangle,
\end{aligned}
$$


where the derivative operator $d_{\alpha}$ acting on the 3 -component vector $\vec{b}_{\beta}$ must be understood as $d_{\alpha} \vec{b}_{\beta}=\partial_{\alpha} \vec{b}_{\beta}+\gamma_{\alpha} \vec{b}_{\beta}$.

These matrices lead to the UV-divergent part of the one-loop contribution to the effective action in Eq. (14), which is given in terms of $\Lambda$ and $Y_{\mu \nu}$, with the latter provided by

$$
\begin{aligned}
Y_{\mu \nu} & =\partial_{\mu} Y_{\nu}-\partial_{\nu} Y_{\mu}+\left[Y_{\mu}, Y_{\nu}\right] \\
& =\left(\begin{array}{cc}
\gamma_{\mu \nu}-\frac{1}{4} B_{\mu \nu} & \frac{1}{2} \vec{b}_{\mu \nu} \\
-\frac{1}{2} \vec{b}_{\mu \nu}^{T} & 0
\end{array}\right), \\
\left(\gamma_{\mu \nu}\right)_{i j} & =-\frac{1}{2}\left\langle\left[\sigma^{i}, \sigma^{j}\right] \Gamma_{\mu \nu}\right\rangle, \\
\Gamma_{\mu \nu} & =\partial_{\mu} \Gamma_{\nu}-\partial_{\nu} \Gamma_{\mu}+\left[\Gamma_{\mu}, \Gamma_{\nu}\right]=\frac{1}{4}\left[u_{\mu}, u_{\nu}\right]-\frac{i}{2} f_{+\mu \nu}, \\
\left(B_{\mu \nu}\right)_{i j} & =\left(\vec{b}_{\mu}\right)_{i}\left(\vec{b}_{\nu}\right)_{j}-\left(\vec{b}_{\nu}\right)_{i}\left(\vec{b}_{\mu}\right)_{j} \\
\left(\vec{b}_{\mu \nu}\right)_{k} & =\left(d_{\mu} \vec{b}_{\nu}-d_{\nu} \vec{b}_{\mu}\right)_{k} .
\end{aligned}
$$

[1] H. Georgi and D. B. Kaplan, Phys. Lett. B 145, 216 (1984).

[2] C. G. Callan, Jr., S. R. Coleman, J. Wess, and B. Zumino, Phys. Rev. 177, 2247 (1969); S. R. Coleman, J. Wess, and B. Zumino, Phys. Rev. 177, 2239 (1969).

[3] R. Contino, arXiv:1005.4269.

[4] J. Gasser and H. Leutwyler, Ann. Phys. (N.Y.) 158 (1984) 142; Nucl. Phys. B250, 465 (1985).

[5] E. E. Jenkins, A. V. Manohar, and M. Trott, J. High Energy Phys. 10 (2013) 087; 01 (2014) 035; R. Alonso, E. E. Jenkins, A. V. Manohar, and M. Trott, J. High Energy Phys. 04 (2014) 159.

[6] S. Weinberg, Physica A 96, 327 (1979).

[7] H. Georgi and A. Manohar, Nucl. Phys. B234, 189 (1984).

[8] R. L. Delgado, A. Dobado, M. J. Herrero, and J. J. SanzCillero, J. High Energy Phys. 07 (2014) 149.

[9] J. Hirn and J. Stern, Eur. Phys. J. C 34, 447 (2004); J. High Energy Phys. 09 (2004) 058.

[10] G. Buchalla and O. Catà, J. High Energy Phys. 07 (2012) 101; G. Buchalla, O. Catà, and C. Krause, Phys. Lett. B 731, 80 (2014); Nucl. Phys. B880, 552 (2014).

[11] A. C. Longhitano, Phys. Rev. D 22, 1166 (1980); Nucl. Phys. B188, 118 (1981); T. Appelquist and C. Bernard, Phys. Rev. D 22, 200 (1980).

[12] M. J. Herrero and E. Ruiz-Morales, Nucl. Phys. B418, 431 (1994); B437, 319 (1995).

[13] M. B. Gavela, K. Kanshin, P. A. N. Machado, and S. Saa, J. High Energy Phys. 03 (2015) 043.

[14] CMS Collaboration, Report No. CMS-NOTE-13-002; Report No. ILC-REPORT-2013-040; Report No. IHEP-CEPCDR-2015-01, http://cepc.ihep.ac.cn/preCDR/volume.html.

[15] R. Alonso, M. B. Gavela, L. Merlo, S. Rigolin, and J. Yepes, Phys. Lett. B 722, 330 (2013); 726, 926 (2013).

[16] A. Azatov, R. Contino, A. Di Iura, and J. Galloway, Phys. Rev. D 88, 075019 (2013).
[17] A. Azatov, C. Grojean, A. Paul, and E. Salvioni, Zh. Eksp. Teor. Fiz. 147, 410 (2015); J. Exp. Theor. Phys. 120, 354 (2015).

[18] A. Azatov, R. Contino, G. Panico, and M. Son, Phys. Rev. D 92, 035001 (2015).

[19] R. L. Delgado, A. Dobado, and F. J. Llanes-Estrada, J. High Energy Phys. 02 (2014) 121; D. Espriu, F. Mescia, and B. Yencho, Phys. Rev. D 88, 055002 (2013).

[20] R. Contino, C. Grojean, M. Moretti, F. Piccinini, and R. Rattazzi, J. High Energy Phys. 05 (2010) 089; A. Azatov, R. Contino, and J. Galloway, J. High Energy Phys. 04 (2012) 127; 04 (2013) 140.

[21] G. F. Giudice, C. Grojean, A. Pomarol, and R. Rattazzi, J. High Energy Phys. 06 (2007) 045.

[22] G. D'Ambrosio, G. F. Giudice, G. Isidori, and A. Strumia, Nucl. Phys. B645, 155 (2002).

[23] G. 't Hooft, Nucl. Phys. B 62, 444 (1973); P. Ramond, Front. Phys. 74, 1 (1989); B. S. DeWitt, Int. Ser. Monogr. Phys. 114, 1 (2003); A. O. Barvinsky and G. A. Vilkovisky, Phys. Rep. 119, 1 (1985); C. Lee, T. Lee, and H. Min, Phys. Rev. D 39, 1681 (1989); R. D. Ball, Phys. Rep. 182, 1 (1989); D. V. Vassilevich, Phys. Rep. 388, 279 (2003).

[24] A. Pich, I. Rosell, and J. J. Sanz-Cillero, Phys. Rev. Lett. 110, 181801 (2013); J. High Energy Phys. 01 (2014) 157.

[25] I. Rosell, P. Ruiz-Femenia, and J. Portoles, J. High Energy Phys. 12 (2005) 020.

[26] J. F. Donoghue, E. Golowich, and B. R. Holstein, Cambridge Monogr. Part. Phys., Nucl. Phys., Cosmol. 2, 1 (1992).

[27] ATLAS Collaboration, Report No. ATLAS-CONF-2014-009; CMS Collaboration, Report No. CMS-PAS-HIG-14-009.

[28] G. Aad et al. (ATLAS Collaboration), Phys. Rev. Lett. 113, 141803 (2014).

[29] S. Chatrchyan et al. (CMS Collaboration), J. High Energy Phys. 07 (2013) 116. 